\title{
Chapter 9 \\ Robust Control of Discrete Linear Repetitive Processes with Parameter Varying Uncertainty
}

\author{
Błażej Cichy, Krzysztof Gałkowski, Eric Rogers and Anton Kummert
}

\begin{abstract}
Repetitive processes propagate information in two independent directions where the duration of one of these is infinite.

They pose control problems that cannot be solved by application of results for other classes of 2D systems. This paper develops robust controller design algorithms for discrete linear processes based on the poly-quadratic stability that produce less conservative results than currently available alternatives.
\end{abstract}

\subsection{Introduction}

Repetitive processes are a distinct class of 2D systems of both system theoretic and applications interest whose unique characteristic is a series of sweeps, termed passes, through a set of dynamics defined over a fixed finite duration known as the pass length. On each pass an output, termed the pass profile, is produced which acts as a

B. Cichy $(\bowtie) \cdot$ K. Gałkowski

Institute of Control and Computation Engineering, University of Zielona Góra, ul. Podgórna 50, 65-246 Zielona Gora, Poland

e-mail: B.Cichy@issi.uz.zgora.pl

K. Gałkowski

e-mail: K.Galkowski@issi.uz.zgora.pl

E. Rogers

School of Electronics and Computer Science, University of Southampton, Southampton, SO17 1BJ, UK

e-mail: etar@ecs.soton.ac.uk

A. Kummert

Faculty of Electrical, Information and Media Engineering, University of Wuppertal,

Rainer-Gruenter-Strasse 21, 42119 Wuppertal, Germany

e-mail: kummert@uni-wuppertal.de 
forcing function on, and hence contributes to, the dynamics of the next pass profile. This, in turn, leads to the unique control problem in that the output sequence of pass profiles can contain oscillations whose amplitude in the pass-to-pass direction.

To introduce a formal definition, let the integer $\alpha<+\infty$ denote the number of samples resulting from sampling the assumed constant pass length at a constant rate. Then in a repetitive process the pass profile $y_{k}(p), 0 \leq p \leq \alpha-1$, generated on pass $k$ acts as a forcing function on, and hence contributes to, the dynamics of the next pass profile $y_{k+1}(p), 0 \leq p \leq \alpha-1, k \geq 0$.

Physical examples of these processes include long-wall coal cutting and metal rolling operations [17]. Also in recent years applications have arisen where adopting a repetitive process setting for analysis has distinct advantages over alternatives. An example of these algorithmic applications is iterative algorithms for solving nonlinear dynamic optimal stabilization problems based on the maximum principle [16] where use of the repetitive process setting provides the basis for the development of highly reliable and efficient iterative solution algorithms. A second example is iterative learning control schemes which form one approach to controlling systems operating in a repetitive (or pass-to-pass) mode with the requirement that a reference trajectory $r(p)$ defined over a finite interval $0 \leq p \leq \alpha-1$ is followed to a high precision-see, for example, [11]. In this case a repetitive process setting for analysis provides a stability theory which, unlike many alternatives, allows for design to meet pass-to-pass error convergence and control of the along the pass dynamics. Also iterative learning control laws designed in this setting have been experimentally verified [10] on a gantry robot with very good correlation between simulation and actually measured performance.

Attempts to analyze repetitive processes using standard (or 1D) systems theory/ algorithms fail (except in a few very restrictive special cases) precisely because such an approach ignores their inherent 2D systems structure, i.e. information propagation occurs from pass-to-pass and along a given pass. Also the initial conditions are reset before the start of each new pass and the structure of these can be somewhat complex. For example, if the pass state initial vector is an explicit function of the pass profile vector at points along the previous pass then this alone can destroy the most basic performance specification of stability. In seeking a rigorous foundation on which to develop a control/estimation/filtering theory for these processes, it is natural to attempt to exploit structural links which exist with other classes of 2D linear systems.

The case of $2 \mathrm{D}$ discrete linear systems recursive in the positive quadrant $(i, j)$ : $i \geq 0, j \geq 0$ (where $i$ and $j$ denote the directions of information propagation) has been the subject of much research effort over the years using, in the main, the well known Roesser and Fornasini Marchesini state-space models (for details on these see, for example, the references given in [17]). More recently, productive research has been reported on $\mathcal{H}_{\infty}$ and $\mathcal{H}_{2}$ approaches to filtering and control law designsee, for example, [3, 19]. (Filtering of this general form is, of course, well established in 1D linear systems theory, see, for example, [8, 13, 18]).

As noted above, the structure of the boundary conditions for linear repetitive processes can cause problems which have no Roesser or Fornasini Marchesini 
state-space model counterparts. Moreover, there are key systems theoretic properties for repetitive processes that have no interpretation in terms of these (and other) 2D systems models. An example here is pass profile controllability [17] that is the physically motivated requirement that there exists a control input sequence which will force the process to produce a pre-specified pass profile on a given pass. This means that the systems theory for other classes of 2D discrete linear systems is very often not applicable to repetitive processes.

As noted above, material, or metal, rolling is one of a number of physically based problems which can be modeled as a linear repetitive process [17]. In this paper, we use a material rolling process as a basis to illustrate the solution we develop to a currently open robust stability and stabilization problem for discrete linear repetitive processes. The design itself can be completed using Linear Matrix Inequalities (LMIs) [4, 12].

In physical application terms, the system or process parameters are most often not known exactly and only some nominal values or admissible intervals are available. Hence, although the nominal process is most often time invariant, the uncertain process can be time-varying. This will be the case here and to solve the problem we generalize previously reported LMI based design algorithms for uncertain discrete linear repetitive processes [5] and other classes of systems $[2,9,15]$ with polytopic uncertainty. These results are based on sufficient, but not necessary, stability conditions.

The use of sufficient but not necessary stability conditions obviously creates a potentially serious problem since the results obtained can be very conservative in the sense that the range over which the admissible parameters can vary is very small. Here we develop substantial new results on how this problem can be overcome. The essential mechanism used is to allow a control law where the entries in the defining matrices explicitly depend on the pass number $k$, and the along the pass variable $p, k \geq 0$ and $0 \leq p \leq \alpha-1$. This is a form of adaptation which allows the control law to follow or track the evolution of any uncertainty present in the model used for control law design and evaluation. In addition to reducing the conservativeness of the design, this will also allow the admissible uncertainty set over which a satisfactory solution exists to be enlarged.

Throughout this chapter, the null matrix and the identity matrix with appropriate dimensions are denoted by 0 and $I$, respectively. Moreover, a real symmetric positive definite matrix, say $N$, is denoted by $N \succ 0$. Next we describe the modeling of the material rolling process used in this paper.

\subsection{Material Rolling as a Linear Repetitive Process}

Material rolling is an extremely common industrial process where, in essence, deformation of the workpiece takes place between two rolls with parallel axes revolving in opposite directions. Consider also the following differential equation model of the metal rolling process shown schematically in Fig. 9.1 whose 
Fig. 9.1 Schematic of material rolling

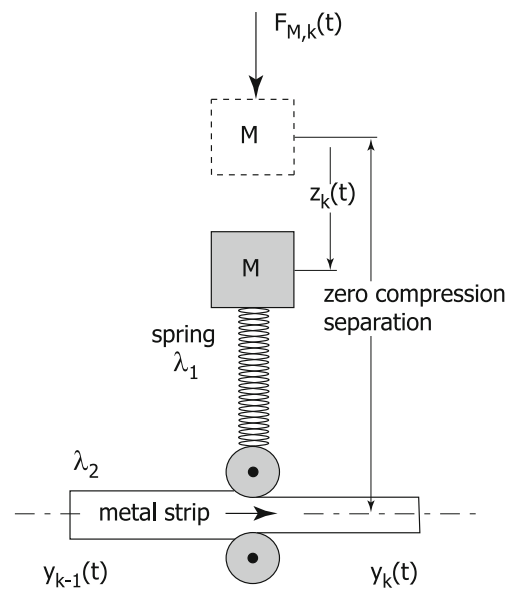

derivation is explained fully in [1] (see also [7] for other repetitive process based analysis of this model)

$$
\ddot{y}_{k}(t)+a_{0} y_{k}(t)+b_{2} \ddot{y}_{k-1}(t)+b_{0} y_{k-1}(t)=c_{0} u_{k}(t)
$$

where

$$
a_{0}=\frac{\lambda_{1} \lambda_{2}}{M\left(\lambda_{1}+\lambda_{2}\right)}, \quad b_{2}=\frac{-\lambda_{2}}{\lambda_{1}+\lambda_{2}}, \quad b_{0}=\frac{-\lambda_{1} \lambda_{2}}{M\left(\lambda_{1}+\lambda_{2}\right)}, \quad c_{0}=\frac{-\lambda_{1}}{M\left(\lambda_{1}+\lambda_{2}\right)}
$$

and $u_{k}(t)=F_{M, k}(t)$. Here $y_{k+1}(t)$ and $y_{k}(t)$ denote the thickness of the material on two successive passes, $M$ is the lumped mass of the roll-gap adjusting mechanism, $\lambda_{1}$ is the stiffness of the adjustment mechanism springs and $\lambda_{2}$ is the hardness of the material strip, and $F_{M, k}(t)$ is the force developed by the motor.

Applying the backward Euler discretization method with sampling period $T$ to (9.1) leads to the following state-space model which is a special case of that for discrete linear repetitive processes

$$
\begin{aligned}
& x_{k+1}(p+1)=A x_{k+1}(p)+B u_{k+1}(p)+B y_{k}(p) \\
& y_{k+1}(p)=C x_{k+1}(p)+D u_{k+1}(p)+D y_{k}(p)
\end{aligned}
$$

where

$$
\begin{array}{rlrl}
A & =\frac{1}{1+a_{0} T^{2}}\left[\begin{array}{cc}
1 & T \\
-a_{0} T & 1
\end{array}\right], & B=\frac{c_{0} T}{1+a_{0} T^{2}}\left[\begin{array}{l}
T \\
1
\end{array}\right] \\
B_{0}=\frac{\left(-b_{0}+a_{0} b_{2}\right) T}{1+a_{0} T^{2}}\left[\begin{array}{l}
T \\
1
\end{array}\right], & C=\frac{1}{1+a_{0} T^{2}}\left[\begin{array}{ll}
1 & T
\end{array}\right] \\
D=\frac{c_{0} T^{2}}{1+a_{0} T^{2}}, & D_{0}=\frac{-b_{2}-b_{0} T^{2}}{1+a_{0} T^{2}}
\end{array}
$$


and

$$
x_{k}(p)=\left[\begin{array}{c}
y_{k+1}(p-1)+b_{2} y_{k}(p-1) \\
\left(y_{k+1}(p-1)+b_{2} y_{k}(p-1)-y_{k+1}(p-2)-b_{2} y_{k}(p-2)\right) T^{-1}
\end{array}\right]
$$

In the general case on pass $k, x_{k+1}(p)$ is the $n \times 1$ current pass state vector $y_{k}(p)$ is the $m \times 1$ pass profile vector and $u_{k}(p)$ is the $l \times 1$ current pass input vector.

To complete the process description it is necessary to specify the initial, or boundary, conditions, that is, the pass state initial vector sequence and the initial pass profile. Here these are taken to be of the form

$$
\begin{aligned}
x_{k+1}(0) & =d_{k+1}, & & k \geq 0 \\
y_{0}(p) & =f(p), & & 0 \leq p \leq \alpha-1
\end{aligned}
$$

where $d_{k+1}$ is an $n \times 1$ vector with constant entries and $f(p)$ is an $m \times 1$ vector whose entries are known functions of $p$.

\subsection{Stability and Stabilization of Discrete Linear Repetitive Processes}

The stability theory [17] for linear repetitive processes is based on an abstract model in a Banach space setting that includes a wide range of such processes as special cases, including those described by (9.2) and (9.3). In terms of their dynamics it is the pass-to-pass coupling (noting again their unique feature) which is critical. This is of the form $y_{k+1}=L_{\alpha} y_{k}$, where $y_{k} \in E_{\alpha}\left(E_{\alpha}\right.$ a Banach space with norm $\|\cdot\|$ ) and $L_{\alpha}$ is a bounded linear operator mapping $E_{\alpha}$ into itself. (In the case of processes described by (9.2) and (9.3), $L_{\alpha}$ is a discrete linear systems convolution operator.)

Asymptotic stability, i.e. bounded-input bounded-output (BIBO) stability over the fixed finite pass length $\alpha>0$, requires the existence of finite real scalars $M_{\alpha}>0$ and $\lambda_{\alpha} \in(0,1)$ such that $\left\|L_{\alpha}^{k}\right\| \leq M_{\alpha} \lambda_{\alpha}^{k}, k \geq 0$, where $\|\cdot\|$ also denotes the induced operator norm. For the processes described by (9.2) and (9.3) it has been shown elsewhere (see, for example, Chap. 3 of Rogers et al. [17]) that this property holds if, and only if, all eigenvalues of the matrix $D_{0}$ have modulus strictly less than unity-written here as $r\left(D_{0}\right)<1$ where $r(\cdot)$ denotes the spectral radius of its matrix argument.

Suppose that $r\left(D_{0}\right)<1$ and the input sequence applied $\left\{u_{k+1}\right\}_{k}$ converges strongly as $k \rightarrow \infty$ (i.e. in the sense of the norm on the underlying function space) to $u_{\infty}$. Then the strong limit $y_{\infty}:=\lim _{k \rightarrow \infty} y_{k}$ is termed the limit profile corresponding to this input sequence and its dynamics are described by 


$$
\begin{aligned}
x_{\infty}(p+1)= & \left(A+B_{0}\left(I-D_{0}\right)^{-1} C\right) x_{\infty}(p) \\
& +\left(B+B_{0}\left(I-D_{0}\right)^{-1} D\right) u_{\infty}(p) \\
y_{\infty}(p)= & \left(I-D_{0}\right)^{-1} C x_{\infty}(p) \\
& +\left(I-D_{0}\right)^{-1} D u_{\infty}(p) \\
x_{\infty}(0)= & d_{\infty}
\end{aligned}
$$

where (again a strong limit) $d_{\infty}:=\lim _{k \rightarrow \infty} d_{k}$. In physical terms, this result states that under asymptotic stability the repetitive dynamics can, after a "sufficiently large" number of passes have elapsed, be replaced by those of a 1D discrete linear system. In particular, this property demands that the amplifying properties of the coupling between successive pass profiles are completely damped out after a sufficiently large number of passes have elapsed. This fact has clear implications in terms of the control of these processes-see [17] for a detailed treatment of this point.

The finite pass length means that the limit profile can have unacceptable along the pass dynamics and, in particular, be unstable in the 1D linear systems sense. A simple example here is the case when $A=-0.5, B=1, B_{0}=0.5+\beta$, $C=1, D=D_{0}=0$, where $\beta>0$ is a real scalar. Hence if $|\beta| \geq 1$ the limit profile is unstable.

If we wish to avoid cases such as this example from arising, one route is to demand the BIBO stability property for any possible value of the pass length (mathematically this can be analyzed by letting $\alpha \rightarrow \infty$ ). This is the stability along the pass property which requires the existence of finite real scalars $M_{\infty}>0$ and $\lambda_{\infty} \in(0,1)$ that are independent of $\alpha$ and are such that $\left\|L_{\alpha}^{k}\right\| \leq M_{\infty} \lambda_{\infty}^{k}, k \geq 0$.

Numerous sets of necessary and sufficient conditions for stability along the pass of (9.2) and (9.3) are known but here it is the following result which is the starting point.

Theorem 1.1 [6]. A discrete linear repetitive process described by (9.2) and (9.3) is stable along the pass if there exist matrices $W_{1} \succ 0$ and $W_{2} \succ 0$ such that

$$
\widehat{A}^{T} W \widehat{A}-W \prec 0
$$

where $W=\operatorname{diag}\left\{W_{1}, W_{2}\right\} \succ 0$, and

$$
\widehat{A}=\left[\begin{array}{ll}
A & B_{0} \\
C & D_{0}
\end{array}\right]
$$

Even though this condition is sufficient but not necessary it forms a basis of control law design via a Lyapunov function interpretation. In particular, follow [14] and introduce the candidate Lyapunov function as

$$
\mathcal{V}(k, p)=x_{k+1}^{T}(p) W_{1} x_{k+1}(p)+y_{k}^{T}(p) W_{2} y_{k}(p)
$$

where $W_{1} \succ 0, W_{2} \succ 0$, with associated increment 


$$
\begin{aligned}
\Delta \mathcal{V}(k, p)= & x_{k+1}^{T}(p+1) W_{1} x_{k+1}(p+1)+y_{k+1}^{T}(p) W_{2} y_{k+1}(p) \\
& -x_{k+1}^{T}(p) W_{1} x_{k+1}(p)-y_{k}^{T}(p) W_{2} y_{k}(p)
\end{aligned}
$$

Then it is easy to show that

$$
\Delta \mathcal{V}(k, p)<0
$$

is equivalent to (9.5).

An extensively analyzed control law for processes described by (9.2) and (9.3) (see, for example, [14]) has the following form over $0 \leq p \leq \alpha-1, k \geq 0$

$$
u_{k+1}(p)=\left[\begin{array}{ll}
K_{1} & K_{2}
\end{array}\right]\left[\begin{array}{c}
x_{k+1}(p) \\
y_{k}(p)
\end{array}\right]
$$

where $K_{1}$ and $K_{2}$ are appropriately dimensioned matrices to be designed. In effect, this control law is composed of a weighted sum of current pass state feedback and feedforward of the previous pass profile.

The LMI of (9.5) extends in a natural manner to the design of (9.10) for stability along the pass but here we will use the approach based on $[2,9,15]$ and first adopted for repetitive processes in [6]. This will prove to be of particular use in the analysis of the case when there is uncertainty associated with the process state-space model.

Theorem 1.2 Suppose that a control law of the form (9.10) is applied to a discrete linear repetitive process described by (9.2) and (9.3). Then the resulting process is stable along the pass if there exist matrices $W=\operatorname{diag}\left\{W_{1}, W_{2}\right\}, W_{1} \succ 0, W_{2} \succ 0$, $G$, and

$$
N=\left[\begin{array}{ll}
\bar{N}_{1} & \bar{N}_{2} \\
\bar{N}_{1} & \bar{N}_{2}
\end{array}\right]
$$

such that

$$
\left[\begin{array}{cc}
-G-G^{T}+W & (\widehat{A} G+\widehat{B} N)^{T} \\
\widehat{A} G+\widehat{B} N & -W
\end{array}\right] \prec 0
$$

If this condition holds, stabilizing $K_{1}$ and $K_{2}$ in the control law (9.10) are given by

$$
K=N G^{-1}
$$

where matrices $K$ and $\widehat{B}$ are given by

$$
K=\left[\begin{array}{ll}
K_{1} & K_{2} \\
K_{1} & K_{2}
\end{array}\right], \quad \widehat{B}=\left[\begin{array}{ll}
B & 0 \\
0 & D
\end{array}\right]
$$


In implementation terms, this control law requires that all elements of the current pass state vector are available for measurement. If this is not true then an observer will required.

Remark 1 The LMIs here are very similar to those known from the literature (see, for example, $[2,9,15])$ for 1D linear systems. This does not, of course, mean that repetitive processes can be analyzed by direct application of existing 1D linear systems theory, merely that in some cases recourse can be made to tools from this latter area. Even then, there are two major differences. The first of these is that the decision matrices must be block-diagonal where the diagonal entries are of dimensions $n \times n$ and $m \times m$ respectively (the first corresponds to the current pass state vector and the second the previous pass profile-see the form of the Lyapunov function). Secondly, even with no uncertainty, the Lyapunov based stability analysis for linear repetitive processes only gives a sufficient condition and hence there is always some conservativeness present.

\subsection{Robust Stability and Stabilization of Discrete Linear Repetitive Processes}

In addition to Theorem 1.2 of the previous section, the design of control laws for discrete linear repetitive processes has been the subject of much research effortsee, for example, [5, 6, 7,14]. Here, we continue the development of this general area by establishing new results related to the practical case where there is possibly large uncertainty associated with the process (state-space model) description. In

particular, we consider the case when the model matrices $\widehat{A}$ and $\widehat{B}$ defined by (9.6) and (9.14) respectively are not precisely known, but belong to a convex bounded (polytope type) uncertain domain denoted here by $\mathcal{D}$. This, in turn, means that any uncertain matrix can be written as a convex combination of the vertices of $\mathcal{D}$ as follows

$$
\begin{aligned}
\mathcal{D}= & \left\{[\widehat{A}(\xi(k, p)), \widehat{B}(\xi(k, p))]:[\widehat{A}(\xi(k, p)), \widehat{B}(\xi(k, p))]=\sum_{i=1}^{v} \xi_{i}(k, p)\left[\widehat{A}_{i}, \widehat{B}_{i}\right],\right. \\
& \left.\sum_{i=1}^{v} \xi_{i}(k, p)=1, \xi_{i}(k, p) \geq 0, k \geq 0,0 \leq p \leq \alpha-1\right\}
\end{aligned}
$$

where $v$ denotes the number of vertices. Note also that the uncertainty here is variable in both independent directions of information propagation, i.e. along the pass ( $p$ direction) and pass-to-pass ( $k$ direction).

At this stage, we can write the following linear parameter dependent statespace model describing the process dynamics 


$$
\begin{aligned}
& x_{k+1}(p+1)=A(\xi(k, p)) x_{k+1}(p)+B(\xi(k, p)) u_{k+1}(p)+B_{0}(\xi(k, p)) y_{k}(p) \\
& y_{k+1}(p)=C(\xi(k, p)) x_{k+1}(p)+D(\xi(k, p)) u_{k+1}(p)+D_{0}(\xi(k, p)) y_{k}(p)
\end{aligned}
$$

Consider also the parameterized candidate Lyapunov function

$$
\mathcal{V}(k, p, \xi(k, p))=x_{k+1}^{T}(p) W_{1}(\xi(k, p)) x_{k+1}(p)+y_{k}^{T}(p) W_{2}(\xi(k, p)) y_{k}(p)
$$

with

$$
\begin{aligned}
& W_{1}(\xi(k, p))=\sum_{i=1}^{v} \xi_{i}(k, p) W_{i 1} \\
& W_{2}(\xi(k, p))=\sum_{i=1}^{v} \xi_{i}(k, p) W_{i 2}
\end{aligned}
$$

and $\mathcal{V}(0,0, \xi(0,0))<\infty$, and associated increment

$$
\begin{aligned}
\Delta \mathcal{V}(k, p, \xi(k, p))= & x_{k+1}^{T}(p+1) W_{1}(\xi(k, p+1)) x_{k+1}(p+1) \\
& +y_{k+1}^{T}(p) W_{2}(\xi(k+1, p)) y_{k+1}(p)-x_{k+1}^{T}(p) W_{1}(\xi(k, p)) x_{k+1}(p) \\
& -y_{k}^{T}(p) W_{2}(\xi(k, p)) y_{k}(p)
\end{aligned}
$$

Then we can define so-called poly-quadratic stability (see [2, 9, 15] for the 1D systems case) as follows.

Definition 1.3 A discrete linear repetitive process described by (9.2) and (9.3) with uncertainty defined by (9.15) and Lyapunov function (9.17) and (9.18) is said to be poly-quadratically stable provided

$$
\Delta \mathcal{V}(k, p, \xi(k, p))<0
$$

for all $k \geq 0,0 \leq p \leq \alpha-1$.

The requirement of (9.20) can be written as

$$
\widehat{A}(\xi(k, p))^{T} \mathcal{W}^{+} \widehat{A}(\xi(k, p))-\mathcal{W} \prec 0
$$

where $\mathcal{W}=\operatorname{diag}\left(\left\{W_{1}(\xi(k, p))\right), W_{2}(\xi(k, p))\right\}$ is defined by (9.18)

$$
\begin{aligned}
\mathcal{W}^{+} & =\operatorname{diag}\left\{W_{1}(\xi(k, p+1)), W_{2}(\xi(k+1, p))\right\} \\
& =\sum_{i=1}^{v} \operatorname{diag}\left\{\xi_{i}(k, p+1) W_{i 1}, \xi_{i}(k+1, p) W_{i 2}\right\} \\
& =\sum_{i=1}^{v} \zeta_{i}(k, p) \operatorname{diag}\left\{W_{i 1}, W_{i 2}\right\}
\end{aligned}
$$

with $\sum_{i=1}^{v} \zeta_{i}(k, p)=1 ; \zeta_{i}(k, p) \geq 0 ; k \geq 0 ; 0 \leq p \leq \alpha-1$. We also require that $\xi_{i}(k, p+1)=\xi_{i}(k+1, p)=\zeta_{i}(k, p)$ and the matrix $\widehat{A}$ of (9.6) in this case becomes 


$$
\widehat{A}(\xi(k, p))=\left[\begin{array}{ll}
A(\xi(k, p)) & B_{0}(\xi(k, p)) \\
C(\xi(k, p)) & D_{0}(\xi(k, p))
\end{array}\right]=\sum_{i=1}^{v} \xi_{i}(k, p) \widehat{A}_{i}
$$

where $\widehat{A}_{i}$ are the polytope vertices (see 9.15).

Remark 2 When

$$
\begin{aligned}
& \operatorname{diag}\left\{W_{1}(\xi(k, p+1)),\right. \\
& \left.\quad W_{2}(\xi(k+1, p))\right\}=\operatorname{diag}\left\{W_{1}(\xi(k, p)), W_{2}(\xi(k, p))\right\}=W
\end{aligned}
$$

and hence $\xi_{i}(k, p)=\zeta_{i}(k, p), i=1, \ldots, v$, poly-quadratic stability reduces to stability along the pass (Theorem 1.1).

The following result (drawing on the work in [2]) aims to minimize the conservativeness present from the use of a sufficient, but not necessary, stability condition.

Theorem 1.4 A discrete linear repetitive process described by (9.2) and (9.3) with uncertainty defined by (9.15) is poly-quadratically stable if there exists blockdiagonal matrices $S_{i} \succ 0, i=1, \ldots, v$, i.e. $S_{i}=\operatorname{diag}\left\{S_{i 1}, S_{i 2}\right\}$, and a matrix $G$ such that

$$
\left[\begin{array}{cc}
G+G^{T}-S_{i} & G^{T} \widehat{A}_{i}^{T} \\
\widehat{A}_{i} G & S_{j}
\end{array}\right] \succ 0
$$

for all $i, j=1, \ldots, v$.

Proof Assume that (9.23) is feasible for all $i, j=1, \ldots, v$. Then

$$
G+G^{T}-S_{i} \succ 0
$$

and, since $G$ is full rank and $S_{i} \succ 0$,

$$
\left(S_{i}-G\right)^{T} S_{i}^{-1}\left(S_{i}-G\right) \succeq 0
$$

or, equivalently,

$$
G^{T} S_{i}^{-1} G \succeq G^{T}+G-S_{i}
$$

Hence if (9.23) holds

$$
\left[\begin{array}{cc}
G^{T} S_{i}^{-1} G & G^{T} \widehat{A}_{i}^{T} \\
\widehat{A}_{i} G & S_{j}
\end{array}\right] \succ 0
$$

or equivalently,

$$
\left[\begin{array}{cc}
G^{T} & 0 \\
0 & S_{j}
\end{array}\right]\left[\begin{array}{cc}
S_{i}^{-1} & \widehat{A}_{i}^{T} S_{j}^{-1} \\
S_{j}^{-1} \widehat{A}_{i} & S_{j}^{-1}
\end{array}\right]\left[\begin{array}{cc}
G & 0 \\
0 & S_{j}
\end{array}\right] \succ 0
$$


Next, introduce the substitutions $W_{i}=S_{i}^{-1}$ and $W_{j}=S_{j}^{-1}$ to obtain

$$
\left[\begin{array}{cc}
W_{i} & \widehat{A}_{i}^{T} W_{j} \\
W_{j} \widehat{A}_{i} & W_{j}
\end{array}\right] \succ 0
$$

for all $i, j=1, \ldots, v$. Further, for each $i$, multiply the corresponding inequalities for $j=1, \ldots, v$ by $\zeta_{j}(k, p)$ and sum over $j$ to obtain

$$
\left[\begin{array}{cc}
W_{i} & \widehat{A}_{i}^{T}\left(\sum_{j=1}^{v} \zeta_{j}(k, p) W_{j}\right) \\
\left(\sum_{j=1}^{v} \zeta_{j}(k, p) W_{j}\right) \widehat{A}_{i} & \sum_{j=1}^{v} \zeta_{j}(k, p) W_{j}
\end{array}\right] \succ 0
$$

Also $\sum_{j=1}^{v} \zeta_{j}(k, p) W_{j}=\mathcal{W}^{+}$and multiplying the resulting inequalities by $\xi_{i}(k, p)$ for $i=1, \ldots, v$, and summing over $i$ gives

$$
\left[\begin{array}{cc}
\sum_{i=1}^{v} \xi_{i}(k, p) W_{i} & \left(\sum_{i=1}^{v} \xi_{i}(k, p) \widehat{A}_{i}^{T}\right) \mathcal{W}^{+} \\
\mathcal{W}^{+}\left(\sum_{i=1}^{v} \xi_{i}(k, p) \widehat{A}_{i}\right) & \mathcal{W}^{+}
\end{array}\right] \succ 0
$$

or, equivalently,

$$
\left[\begin{array}{cc}
\mathcal{W} & \widehat{A}^{T}(\xi(k, p)) \mathcal{W}^{+} \\
\mathcal{W}^{+} \widehat{A}(\xi(k, p)) & \mathcal{W}^{+}
\end{array}\right] \succ 0
$$

Finally, applying Definition 1.3, followed by an obvious application of the Schur's complement formula, to (9.24) gives

$$
\mathcal{W}-\widehat{A}(\xi(k, p))^{T} \mathcal{W}^{+} \widehat{A}(\xi(k, p)) \succ 0
$$

which is equivalent to (9.21) and the proof is complete.

Remark 3 Recall that the diagonal structure of $S_{i}$ in this last result arises directly from the stability theory for discrete linear repetitive processes. As noted previously, this leads to only sufficient conditions and hence some conservativeness can be present which is, however, lower than if the matrices $G_{i}$ were also taken as block-diagonal (these matrices only relate to the corresponding LMI construction).

With the control law (9.10) applied, (9.23) becomes

$$
\left[\begin{array}{cc}
G+G^{T}-S_{i} & G^{T}\left(\widehat{A}_{i}+\widehat{B}_{i} K\right)^{T} \\
\left(\widehat{A}_{i}+\widehat{B}_{i} K\right) G & S_{j}
\end{array}\right] \succ 0
$$

where $K$ is defined in (9.14), and the following result now gives a sufficient condition for the existence of a poly-quadratically stabilizing control law of the form (9.10). 
Theorem 1.5 Suppose that a control law of the form (9.10) is applied to a discrete linear repetitive process described by (9.2) and (9.3) with uncertainty defined by (9.15). Then the resulting process is poly-quadratically stabilizable if there exist matrices $S_{i} \succ 0, i=1, \ldots, v$, i.e. $S_{i}=\operatorname{diag}\left\{S_{i 1}, S_{i 2}\right\}, G$, and $N$ (defined by (9.11)) such that

$$
\left[\begin{array}{cc}
G+G^{T}-S_{i} & G^{T} \widehat{A}_{i}^{T}+N^{T} \widehat{B}_{i}^{T} \\
\widehat{A}_{i} G+\widehat{B}_{i} N & S_{j}
\end{array}\right] \succ 0
$$

for all $i, j=1, \ldots, v$. If this condition holds then stabilizing $K_{1}$ and $K_{2}$ in the control law are given by (9.10) with

$$
K=N G^{-1}
$$

where $K$ was defined in (9.14).

Proof This result follows immediately from (9.26), on setting $K G=N$.

In the next section we consider parameter variable control laws in an attempt to reduce the level of conservativeness associated with the results so far.

\subsection{A Parameter Variable Control Law}

To enlarge the admissible uncertainty range for which stabilization is still possible (and hence reduce conservativeness), this section considers a parameter variable control law of the form

$$
\begin{aligned}
u_{k+1}(p) & =K(\xi(k, p))\left[\begin{array}{c}
x_{k+1}(p) \\
y_{k}(p)
\end{array}\right]=\left[\begin{array}{ll}
K_{1}(\xi(k, p)) & K_{2}(\xi(k, p))
\end{array}\right]\left[\begin{array}{c}
x_{k+1}(p) \\
y_{k}(p)
\end{array}\right] \\
& =\sum_{i=1}^{v} \xi_{i}(k, p)\left[\begin{array}{ll}
K_{i 1} & K_{i 2}
\end{array}\right]\left[\begin{array}{c}
x_{k+1}(p) \\
y_{k}(p)
\end{array}\right]
\end{aligned}
$$

over $0 \leq p \leq \alpha-1, k \geq 0$ where the designed matrix $K(\xi(k, p))$ contains uncertainty as defined in (9.15). In effect, this control law is composed of the weighted sum of current pass state feedback and feedforward of the previous pass profile and we have the following result (which can be interpreted as the generalization to the repetitive process case of a well known result [2]).

Theorem 1.6 A discrete linear repetitive process described by (9.2) and (9.3) with uncertainty structure of the form (9.15) is poly-quadratically stable, if there exist matrices $S_{i} \succ 0$, i.e. $S_{i}=\operatorname{diag}\left\{S_{i 1}, S_{i 2}\right\} \succ 0$, and $G_{i}, i=1, \ldots v$, such that

$$
\left[\begin{array}{cc}
G_{i}+G_{i}^{T}-S_{i} & G_{i}^{T} \widehat{A}_{i}^{T} \\
\widehat{A}_{i} G_{i} & S_{j}
\end{array}\right] \succ 0
$$

for all $i, j=1, \ldots, v$. 
Proof Assume that (9.30) is feasible for all $i, j=1, \ldots, v$. Then

$$
G_{i}+G_{i}^{T}-S_{i} \succ 0
$$

and, since $G_{i}$ is full rank and $S_{i} \succ 0$

$$
\left(S_{i}-G\right)^{T} S_{i}^{-1}\left(S_{i}-G_{i}\right) \succeq 0
$$

or, equivalently,

$$
G_{i}^{T} S_{i}^{-1} G_{i} \succeq G_{i}^{T}+G_{i}-S_{i}
$$

Hence if (9.30) holds

$$
\left[\begin{array}{cc}
G_{i}^{T} S_{i}^{-1} G_{i} & G_{i}^{T} \widehat{A}_{i}^{T} \\
\widehat{A}_{i} G_{i} & S_{j}
\end{array}\right] \succ 0
$$

or equivalently,

$$
\left[\begin{array}{cc}
G_{i}^{T} & 0 \\
0 & S_{j}
\end{array}\right]\left[\begin{array}{cc}
S_{i}^{-1} & \widehat{A}_{i}^{T} S_{j}^{-1} \\
S_{j}^{-1} \widehat{A}_{i} & S_{j}^{-1}
\end{array}\right]\left[\begin{array}{cc}
G_{i} & 0 \\
0 & S_{j}
\end{array}\right] \succ 0
$$

Next, introduce the substitutions $W_{i}=S_{i}^{-1}$ and $W_{j}=S_{j}^{-1}$ to obtain

$$
\left[\begin{array}{cc}
W_{i} & \widehat{A}_{i}^{T} W_{j} \\
W_{j} \widehat{A}_{i} & W_{j}
\end{array}\right] \succ 0
$$

for all $i, j=1, \ldots, v$. Further, for each $i$, multiply the corresponding inequalities for $j=1, \ldots, v$ by $\zeta_{j}(k, p)$ and sum over $j$ to obtain

$$
\left[\begin{array}{cc}
W_{i} & \widehat{A}_{i}^{T}\left(\sum_{j=1}^{v} \zeta_{j}(k, p) W_{j}\right) \\
\left(\sum_{j=1}^{v} \zeta_{j}(k, p) W_{j}\right) \widehat{A}_{i} & \sum_{j=1}^{v} \zeta_{j}(k, p) W_{j}
\end{array}\right] \succ 0
$$

Also $\sum_{j=1}^{v} \zeta_{j}(k, p) W_{j}=\mathcal{W}^{+}$and multiplying the resulting inequalities by $\xi_{i}(k, p)$ for $i=1, \ldots, v$, and summing over $i$ gives

$$
\left[\begin{array}{cc}
\sum_{i=1}^{v} \xi_{i}(k, p) W_{i} & \left(\sum_{i=1}^{v} \xi_{i}(k, p) \widehat{A}_{i}^{T}\right) \mathcal{W}^{+} \\
\mathcal{W}^{+}\left(\sum_{i=1}^{v} \xi_{i}(k, p) \widehat{A}_{i}\right) & \mathcal{W}^{+}
\end{array}\right] \succ 0
$$

or, equivalently,

$$
\left[\begin{array}{cc}
\mathcal{W} & \widehat{A}^{T}(\xi(k, p)) \mathcal{W}^{+} \\
\mathcal{W}^{+} \widehat{A}(\xi(k, p)) & \mathcal{W}^{+}
\end{array}\right] \succ 0
$$


Applying Definition 1.3, followed by an obvious application of the Schur's complement formula, to (9.31) now gives

$$
\mathcal{W}-\widehat{A}(\xi(k, p))^{T} \mathcal{W}^{+} \widehat{A}(\xi(k, p)) \succ 0
$$

which is equivalent to (9.21) and the proof is complete.

Applying the control law (9.29) to (9.30) gives

$$
\left[\begin{array}{cc}
G_{i}+G_{i}^{T}-S_{i} & G_{i}^{T}\left(\widehat{A}_{i}+\widehat{B}_{i} K_{i}\right)^{T} \\
\left(\widehat{A}_{i}+\widehat{B}_{i} K_{i}\right) G_{i} & S_{j}
\end{array}\right] \succ 0
$$

where the matrix $K_{i}, i=1, \ldots, v$, is given by

$$
K_{i}=\left[\begin{array}{ll}
K_{i 1} & K_{i 2} \\
K_{i 1} & K_{i 2}
\end{array}\right]
$$

The following result now gives a condition sufficient for the existence of a polyquadratically stabilizing control law of the form (9.29) together with a design algorithm.

Theorem 1.7 Suppose that a control law of the form (9.29) is applied to a discrete linear repetitive process described by (9.2) with uncertainty of the form (9.15). Then the resulting process is poly-quadratically stable if there exist block-diagonal matrices $S_{i} \succ 0$, i.e. $S_{i}=\operatorname{diag}\left\{S_{i 1}, S_{i 2}\right\} \succ 0, G_{i}$, and

$$
N_{i}=\left[\begin{array}{ll}
N_{i 1} & N_{i 2} \\
N_{i 1} & N_{i 2}
\end{array}\right]
$$

$i=1, \ldots, v$, such that

$$
\left[\begin{array}{cc}
G_{i}+G_{i}^{T}-S_{i} & G_{i}^{T} \widehat{A}_{i}^{T}+N_{i}^{T} \widehat{B}_{i}^{T} \\
\widehat{A}_{i} G_{i}+\widehat{B}_{i} N_{i} & S_{j}
\end{array}\right] \succ 0
$$

for all $i, j=1, \ldots, v$. If these conditions hold, the vertex matrices $K_{i 1}$ and $K_{i 2}$ in the stabilizing control law of (9.29) are given by

$$
K_{i}=N_{i} G_{i}^{-1}
$$

where $K_{i}$ is defined in (9.34).

Proof This follows immediately from (9.33) on setting $K_{i} G_{i}=N_{i}$.

Theorem 1.7 and (9.29) provides the setting for the construction of the control law considered to stabilize the process with much wider uncertainty, or variability 
domains, than alternatives. However, the final control law is still not available at this stage as only the vertex values of the required matrices are known. To complete the task, we need to know the exact parameters $\xi_{i}(k, p), k \geq 0,0 \leq p \leq \alpha-1$, and $i=1, \ldots, v$, which can be recovered from the process dynamics by using the Matlab function "fmincon" (or any equivalent algorithm) to solve the following problem:

Determine $\xi_{i}(k, p) \in \mathbf{R}^{+}, i=1, \ldots, v$ such that

$$
\sum_{i=1}^{v} \xi_{i}(k, p) V_{i}=P(k, p)
$$

where

$$
\sum_{i=1}^{v} \xi_{i}(k, p)=1, \xi_{i}(k, p) \geq 0, k \geq 0,0 \leq p \leq \alpha-1
$$

and

$$
P(k, p)=\left\{A(k, p), B(k, p), B_{0}(k, p), C(k, p), D(k, p), D_{0}(k, p)\right\}
$$

where $V_{i}$ denotes the convex domain vertices and $P(k, p)$ the process state-space model matrix which is assumed to lie in the polytope constructed from them. (This construction will be explained in detail for the material rolling example considered in the next section).

Given $\xi_{i}(k, p), k \geq 0,0 \leq p \leq \alpha-1, i=1, \ldots, v$, and the vertex matrices of the control law (9.29) from (9.37), we can complete the design using

$$
K(\xi(k, p))=\left[K_{1}(\xi(k, p)) \quad K_{2}(\xi(k, p))\right]=\sum_{i=1}^{v} \xi_{i}(k, p)\left[\begin{array}{ll}
K_{i 1} & K_{i 2}
\end{array}\right]
$$

\subsection{Application to Material Rolling}

In this section we illustrate Theorem 1.7 by application to the material rolling model of Sect. 9.2 when the model parameters $\lambda_{1}, \lambda_{2}$ are uncertain and the rest of parameters (i.e. $T$ and $M$ ) are constant. Also we take $T=0.2 \mathrm{~s}, M=100 \mathrm{~kg}$, and assume that the model parameters $\lambda_{1}$ and $\lambda_{2}$ satisfy

$$
\lambda_{1} \in\left[\underline{\lambda_{1}}, \overline{\lambda_{1}}\right]=[0.216,0.984], \quad \lambda_{2} \in\left[\underline{\lambda_{2}}, \overline{\lambda_{2}}\right]=[0.72,3.28]
$$

Note first that a control law of the form of Theorem 1.5 can be computed in this case but requires that $\lambda_{1}$ and $\lambda_{2}$ satisfy 


$$
\lambda_{1} \in\left[\underline{\lambda_{1}}, \overline{\lambda_{1}}\right]=[0.228,0.978], \quad \lambda_{2} \in\left[\underline{\lambda_{2}}, \overline{\lambda_{2}}\right]=[0.76,3.26]
$$

i.e. this design comes at the price of a more restrictive range for the values of $\lambda_{1}$ and $\lambda_{2}$.

The fact that this problem has two variables that can vary means that there are four uncertainty domain vertices. Also the uncertainty domain for the process matrices is easily verified as convex with vertices as follows vertex 1

$$
\begin{array}{lll}
A=\left[\begin{array}{cc}
0.9377 & 187.5361 \\
-3.116 \cdot 10^{-4} & 0.9377
\end{array}\right], & B=\left[\begin{array}{cl}
-0.0866 \\
-4.3278 \cdot 10^{-4}
\end{array}\right], & B_{0}=\left[\begin{array}{c}
0.0144 \\
7.1907 \cdot 10^{-5}
\end{array}\right] \\
C=\left[\begin{array}{ll}
0.9377 & 187.5361
\end{array}\right], & D=-0.0866, & D_{0}=0.7836
\end{array}
$$

vertex 2

$$
\begin{array}{lll}
A=\left[\begin{array}{cc}
0.7676 & 153.5191 \\
-1.162 \cdot 10^{-3} & 0.7676
\end{array}\right], & B=\left[\begin{array}{c}
-0.0709 \\
-3.5427 \cdot 10^{-4}
\end{array}\right], & B_{0}=\left[\begin{array}{c}
0.0536 \\
2.6816 \cdot 10^{-4}
\end{array}\right] \\
C=\left[\begin{array}{ll}
0.7676 & 153.5191
\end{array}\right], & D=-0.0709, & D_{0}=0.8229
\end{array}
$$

vertex 3

$$
\begin{array}{lll}
A=\left[\begin{array}{cc}
0.8574 & 171.481 \\
-7.1297 \cdot 10^{-4} & 0.8574
\end{array}\right], & B=\left[\begin{array}{c}
-0.198 \\
-9.9024 \cdot 10^{-4}
\end{array}\right], & B_{0}=\left[\begin{array}{c}
0.0823 \\
4.1172 \cdot 10^{-4}
\end{array}\right] \\
C=\left[\begin{array}{ll}
0.8574 & 171.481
\end{array}\right], & D=-0.198, & D_{0}=0.5049
\end{array}
$$

vertex 4

$$
\begin{array}{lll}
A=\left[\begin{array}{cc}
0.925 & 185.0033 \\
-3.7492 \cdot 10^{-4} & 0.925
\end{array}\right], & B=\left[\begin{array}{c}
-0.0229 \\
-1.143 \cdot 10^{-4}
\end{array}\right], & B_{0}=\left[\begin{array}{c}
4.6328 \cdot 10^{-3} \\
2.32 \cdot 10^{-5}
\end{array}\right] \\
C=\left[\begin{array}{ll}
0.925185 .0033
\end{array}\right], & D=-0.0229, & D_{0}=0.9428
\end{array}
$$

The parameters $\lambda_{1}, \lambda_{2}$ vary with $k$ and $p$ and, since they are different on each pass, we denote them by $\lambda_{1}(k, p)$ and $\lambda_{2}(k, p)$, respectively. Also they are assumed to lie in the fixed intervals given by (9.41) as shown in Fig. 9.2.

Theorem 1.7 in this case can provide a variety of possible control laws but it is very difficult to select the one which will also satisfy the performance specifications. To overcome this problem, we use the following corollary of Theorem 1.7.

Corollary 1.8 Suppose that a control law of the form (9.29) is applied to a discrete linear repetitive process described by (9.2) and (9.3) with uncertainty of the form (9.15). Then the resulting process is poly-quadratically stable if there exist block-diagonal matrices $S_{i}$, i.e. $S_{i}=\operatorname{diag}\left\{S_{i 1}, S_{i 2}\right\} \succ 0$, and matrices $G_{i}$ and $N_{i}, i=1, \ldots, v$, (where $N_{i}$ is defined in (9.35)), such that the following convex optimization problem has a solution 
(a)

$\lambda_{1}(k, p)$; number of passes: 25 ; number of points: 25 range: passes $[0,24]$; points: $[0,24]$

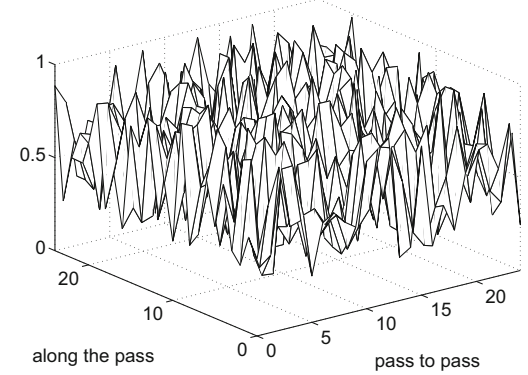
(b) $\lambda_{2}(\mathrm{k}, \mathrm{p})$; number of passes: 25 ; number of points: 25
range: passes $[0,24]$; points: $[0,24]$

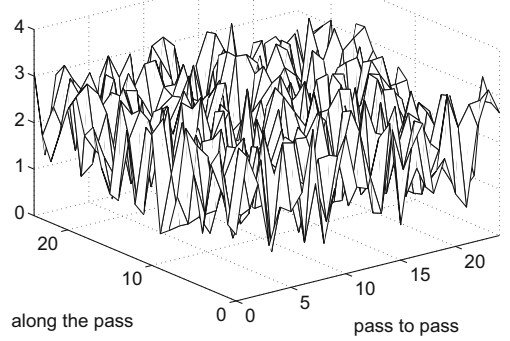

Fig. 9.2 a Values for $\lambda_{1}(k, p)$; b values for $\lambda_{2}(k, p)$

$$
\begin{aligned}
& \operatorname{maximize} \quad F=\sum_{i=1}^{v} \operatorname{trace}\left(G_{i}\right) \\
& \text { subject to } \\
& {\left[\begin{array}{cc}
G_{i}+G_{i}^{T}-S_{i} & G_{i}^{T} \widehat{A}_{i}^{T}+N_{i}^{T} \widehat{B}_{i}^{T} \\
\widehat{A}_{i} G_{i}+\widehat{B}_{i} N_{i} & S_{j}
\end{array}\right] \succ 0}
\end{aligned}
$$

Also we require that the matrices $G_{i}$ and $S_{i}$ are diagonal for all $i=1, \ldots, v$. The control law matrix vertices are obtained as in Theorem 1.7.

For the case considered here, this last result gives the following control law matrix vertices

$$
\begin{array}{lll}
K_{11}=\left[\begin{array}{ll}
3.4741 & 2166.7
\end{array}\right], & K_{12}=0.0873 \\
K_{21}=\left[\begin{array}{ll}
4.4876 & 2166.7
\end{array}\right], & K_{22}=0.1504 \\
K_{31}=\left[\begin{array}{ll}
2.0452 & 865.8534
\end{array}\right], & K_{32}=0.0818 \\
K_{41}=\left[\begin{array}{ll}
10.6 & 8092.6
\end{array}\right], & K_{42}=0.215
\end{array}
$$

Now we are in a position to develop the procedure by which the variable control law matrices for a given $k$ and point $p$ are derived. Consider the matrix of (9.39) for given $T, M, \lambda_{1}(k, p)$ and $\lambda_{2}(k, p)$, where $A(k, p), \ldots, D_{0}(k, p)$ denote the system matrices $A, \ldots, D_{0}$ computed for $T=t, M=m$, and variable parameters $\lambda_{1}=$ $\lambda_{1}(k, p)$ and $\lambda_{2}=\lambda_{2}(k, p)$ from (9.1)-(9.2) at given $k, p$. Having obtained $P(k, p)$ for $k, p$ we can now recover from (9.38) (by using an algorithm based on the Matlab function "fmincon") the underling parameters $\xi_{i}(k, p), k \geq 0,0 \leq p \leq \alpha-1$, and $i=1, \ldots, v$. Then, since the matrix vertices at $(k, p)$ are known, it is a simple task to calculate the variable control law matrices at $(k, p)$ using (9.40). Next, we go to $p+1$ if $p<\alpha-1$ with $k$ unchanged or if $p=\alpha-1$ we go to $k+1$ and set $p=0$, and so on.

Without control action, the process here is unstable along the pass. Suppose also that the task is to reduce the thickness of the workpiece by one unit in the case when $\alpha=25$. Then, since the process dynamics are assumed to be linear, we can take the boundary conditions to be $x_{k+1}(p)=0, k \geq 0$, and $y_{0}(p)=1,0 \leq p \leq 24$. 
(a) $y_{k}^{1 / 1}(p)$; number of passes: 25 ; number of points: 25 range: passes $[0,24]$; points: $[0,24]$

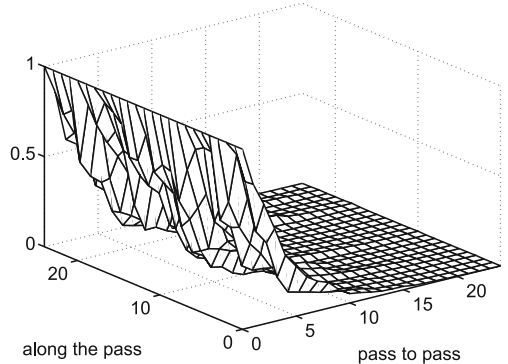

(b)

$u_{k}^{1 / 1}(p)$; number of passes: 25 ; number of points: 25 range: passes $[0,24]$; points: $[0,24]$

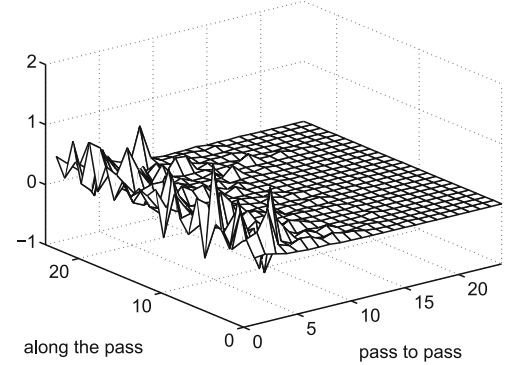

Fig. 9.3 a The process pass profile sequence for the controlled process; $\mathbf{b}$ the control input sequence

Hence as $k$ increases the sequence of pass profiles should approach zero. This is confirmed by the plot of Fig. 9.3a and there are two other major issues which need to be considered. These are the transient behavior in both $p$ and $k$ and the magnitude of the control input signal respectively. If the transient performance is not acceptable, the only option is to return and attempt to tune the design. For the second, Fig. 9.3b shows the control input sequence required to apply the control law in this case. If this is unacceptable, then further development is required and again this is left as a subject for further work-the result here shows that the control action is bounded and hence baseline acceptable.

Finally, note that the control action energy required (maximum absolute value of the control signal) is much lower than that arising when Corollary 1.8 is not applied. Now, however, more passes must be completed before the control objectives are met.

\subsection{Conclusions}

This chapter has focused on the control of discrete linear repetitive processes in the presence of uncertainty in the state-space model used for control law design. A review of previous results in this area leads to the conclusion that the design of practically relevant control laws is possible, but the uncertainty model which has been used to obtain such results may be restrictive in the sense that there is little margin for tuning the control law matrices to obtain stability plus desired performance. The main objective in the work reported in this chapter is to develop control laws which vary in both the pass number and along the pass variables. This has been achieved by allowing the control law matrices to vary with both the pass number and the along the pass variable. The result is an algorithm for basic selection of the control law matrices without destroying the static nature of the control law. 


\section{References}

1. Agathoklis P, Foda S (1989) Stability and the matrix Lyapunov equation for delay differential systems. Int J Control 49(2):417-432

2. Daafouz J, Bernussou J (2001) Parameter dependent Lyapunov functions for discrete time systems with time varying parametric uncertainties. Syst Control Lett 43:355-359

3. Du C, Xie L (2002) $H_{\infty}$ Control and Filtering of Two-dimensional Systems, volume 278 of Lecture notes in control and information sciences. Springer, Berlin

4. Gahinet P, Nemirowski A, Laub AJ, Chilali M (1995) LMI Control Toolbox for use with MATLAB. The Mathworks Partner Series. The MathWorks Inc

5. Gałkowski K, Rogers E, Xu S, Lam J, Owens DH (2002) LMIs-a fundamental tool in analysis and controller design for discrete linear repetitive processes. IEEE Trans Circuits Syst I: Fundam Theory Appl 49(6):768-778

6. Gałkowski K, Lam J, Rogers E, Xu S, Sulikowski B, Paszke W, Owens DH (2003) LMI based stability analisys and robust controller design for discrete linear repetitive processes. Int J Robust Nonlinear Control 13:1195-1211

7. Gałkowski K, Rogers E, Paszke W, Owens DH (2003) Linear repetitive process control theory applied to a physical example. Appl Math Comp Sci 13(1):87-99

8. Geromel JC, de Oliveira MC (2001) $H_{2}$ and $H_{\infty}$ robust filtering for convex bounded uncertain systems. IEEE Trans Autom Control 46(1):100-107

9. Geromel JC, de Oliveira MC, Hsu L (1998) LMI characterization of structural and robust stability. Linear Algebra Appl 285:69-80

10. Hladowski L, Gałkowski K, Cai Z, Rogers E, Freeman CT, Lewin PL (2008) A 2D systems approach to iterative learning control with experimental verification. In: Proceedings of 17th IFAC World Congress, pp 2832-2837

11. Moore KL, Chen YQ, Bahl V (2005) Monotonically convergent iterative learning control for linear discrete-time systems. Automatica 41(9):1529-1537

12. Nesterov Y, Nemirovskii A (1994) Interior-point polynomial algorithms in convex programing, vol 13 of SIAM studies in applied mathematics. SIAM, Philadelphia

13. de Oliveira MC, Bernussou J, Geromel J (1999) A new discrete-time robust stability condition. Syst Control Lett 37:261-265

14. Paszke W, Gałkowski K, Rogers E, Owens DH (2006) $H_{\infty}$ and guaranteed cost control of discrete linear repetitive processes. Linear Algebra Appl 412:93-131

15. Peaucelle D, Arzelier D, Bachelier O, Bernussou J (2000) A new robust D-stability condition for polytopic uncertainty. Syst Cont Lett 40:21-30

16. Roberts PD (2002) Two-dimensional analysis of an iterative nonlinear optimal control problem. IEEE Trans Circuits Syst I: Fundam Theory Appl 48(6):872-878

17. Rogers E, Gałkowski K, Owens DH (2007) Control systems theory and applications for linear repetitive processes, volume 349 of Lecture Notes in Control and Information Sciences. Springer, Heidelberg

18. de Souza CE, Trofino A (1999) Advances in linear matrix inequality methods in control: advances in design and control. In: Recent advances on linear matrix inequalities in control. Philadelphia. SIAM, pp 175-185

19. Tuan HD, Apkarian P, Nguyen TQ (2002) Robust mixed $H_{2} / H_{\infty}$ filtering of 2-D systems. IEEE Trans Signal Process 50(7):1759-1771 